\title{
Impact Analysis of Controllable Home Appliances and EVs on Neighbourhood Level Network with Smart Control
}

\author{
Di Wu${ }^{1}$, Haibo Zeng ${ }^{2}$, Benoit Boulet ${ }^{1}$ \\ ${ }^{1}$ Department of Electrical and Computer Engineering, McGill University, QC H3A OG4, Montreal, Quebec, Canada, \\ \{di.wu5@mail.mcgill.ca, benoit.boulet@mcgill.ca\} \\ ${ }^{2}$ Department of Electrical and Computer Engineering, Virginia Tech, VA 24061, Blacksburg, US, hbzeng@vt.edu
}

\begin{abstract}
With the advance of manufacturing technologies and increasing attention on environment protection, electric vehicles (EVs) are expected to see a fast development. However, large adoption of EVs may impose a high power demand on the grid and may affect the network infrastructures. Controlled power consumption for EV charging and home appliances could help to reduce such pressures on power systems. This paper aims at analyzing the impacts of integration control for EV charging, controllable home appliances, vehicle-to-home (V2H) and home based batteries on neighbourhood level network. Simulation results are presented for Nissan Leaf and Tesla Model S.
\end{abstract}

Keywords: charging, energy consumption, EV, smart, V2G

\section{Introduction}

Road transportation contributes 27\% of total carbon dioxide emission for United States in 2013 [1]. To reduce the greenhouse gas emission as well as dependency on oil, incentives and policies have been proposed in many countries to encourage the transportation electrification [2]. Hybrid electric vehicles (HEVs), battery electric vehicles (BEVs) are increasing quickly in the world vehicle market. Several countries proposed related policies to promote the development of EVs. It is expected that in 2040 two thirds of light-duty vehicles in USA ought to be EVs. The production of EVs in Germany should reach five millions in 2030 [3].

However, the charging of these EVs as a whole will cause a high power demand on the power system. When considering that most of the EVs will be charged at home [4] and the charging time coincides with the residential power consumption, then the charging power issues will be more severe for the neighbourhood level network. Without coordination, EV charging may cause significant negative impacts on the power grid, especially for those high EV adoption areas. Currently there are mainly two kinds of control strategies: centralized control strategy and decentralized control strategy. For the centralized control strategy, there will be a central controller to manage the charging or discharging of all the vehicles. The customers need to give out the charging control for their own EVs for centralized control. As for decentralized control, the charging and discharging could be controlled locally by home based energy management systems.

The vehicle-to-grid (V2G) capability of the EVs means that the EV batteries could be treated as distributed energy storage. The integration of large population of EVs may pose a significant impact on the power grid [5]. Vehicle-to-home (V2H) is a subset of vehicle-to-grid [6]. For V2H, the energy stored in EVs could be discharged back to homes for residential power consumption [7]. In the neighbourhood 
level network, with $\mathrm{V} 2 \mathrm{H}$, the energy stored in EV batteries could be discharged back and serve as energy storage to counter the uncertainty of renewable energy and varying electricity price.

There are two kinds of power consumption for the neighbourhood level network: EV charging power consumption and base load power consumption which describes the power consumption consumed by home appliances. Currently, the home appliances consumption is mostly controlled with simple local controller. And not all of these home appliances could be controlled automatically [8]. In [9], the author proposed a control strategy for home energy management system which consider the EVs, home appliances, ESS, and renewable energy. In [10], a control strategy is proposed with considering the varying electricity price. The impacts of electric vehicles charging on power network have been discussed in [11], [12]. In [11], it is stated that even a small EV penetration would cause significant power increase. In [12], It is shown that without controlling, EV charging could accelerate the transformer ageing significantly.

Unlike previous work, in this paper, we focus on analyzing the impacts for integration control of home appliances, EVs, home batteries on the neighbourhood level network. Simulation results are presented with actual power consumption data. In this paper, we propose a centralized control framework for power consumption of the neighbourhood level network. We assume the central controller could access all the information of the base load information and customers driving behaviors (arrival time, departure time, and arrival battery state of charge). This paper is organized as follows: The system models, including base load power consumption, electric vehicle, neighbourhood level network and time-of-use (TOU) price structure are demonstrated in Section 2. In Section 3, a control framework to minimize the peak power consumption is proposed. The simulation results are presented in Section 4. The paper is concluded in Section 5. Future work is also discussed in this section.

\section{System Models}

To analyze the impacts for integration control of EVs, controllable home appliances and home based batteries on neighbourhood level network, four system models are discussed: base load power consumption, electric vehicles, neighbourhood level network and time-of-use price structure.

\subsection{Base load power consumption}

In this paper, we consider that there are three categories of power consumption in total: electric vehicles charging power consumption $\left(p_{c}\right)$, controllable base load power consumption $\left(p_{b c}\right)$, and uncontrollable base load power consumption $\left(p_{b u}\right)$. Base load means all power consumption in the neighbourhood level network except the EV charging power consumption. Base load power consumption for two typical days in a neighbourhood level network in Ottawa are shown in Figure 1. We can see that the power consumption in winter day is a little lower than that in summer day and there will be valley hours for both summer and winter days (In winter, gas is used for heating).

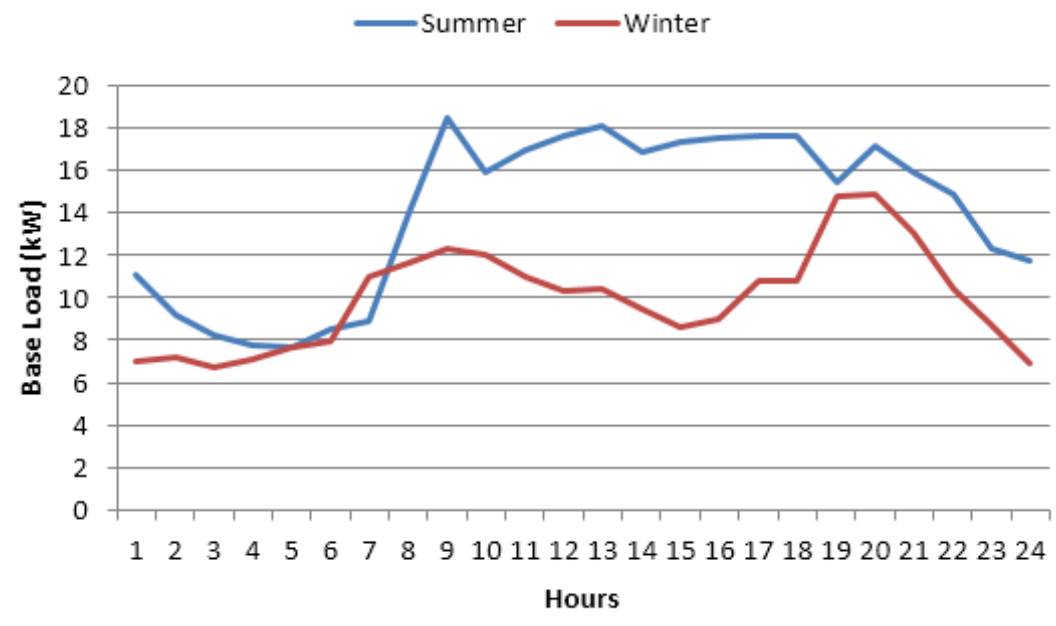

Figure 1: Base load power consumption 


\subsection{Electric vehicles}

The power consumption of electric vehicles can be viewed as a kind of flexible and uncertain power consumption. The uncertainties of EV charging for: arriving time, arriving battery state of charge, and leaving time should be considered for modeling the EV charging power. Table 1 shows the driving distance distributions for the customers. We can see that in the weekend people have a high probability to take a long dirve.

Table 1: Driving distance distribution

\begin{tabular}{cccccc}
\hline Driving Distance (KM) & $0-9$ & $10-24$ & $25-49$ & $50-99$ & $100+$ \\
\hline Weekday & $27 \%$ & $33 \%$ & $25 \%$ & $9 \%$ & $4 \%$ \\
\hline weekend & $18 \%$ & $32 \%$ & $18 \%$ & $18 \%$ & $11 \%$ \\
\hline
\end{tabular}

The EV battery capacity and rated charging power will influence the EV charging demand. The specifications for two kinds of EVs are shown in Table 2. In this paper, the continuous charging is adopted which means that EVs could be charged at a continuous rate from zero to the maximal rated charging power (the charging power will be fixed for one time slot). In this paper, centralized control is adopted which means that the central controller could control the charging and discharging for all the EVs.

Table 2: Specifications for Tesla Model S and Honda Fit

\begin{tabular}{|c|c|c|}
\hline Car Models & Battery Capacity $(\mathrm{kWh})$ & Rated Charging Power(kW) \\
\hline Nissan Leaf [13] & 24 & 6 \\
\hline Tesla Model S [14] & 60 & 10 \\
\hline
\end{tabular}

\section{3 neighbourhood level network}

Figure 2 shows a typical neighbourhood level network. One transformer is responsible for providing energy for the neighbourhood level network. In Figure 2, we assume that some homes are integrated with Tesla Power Wall. It shows that for a typical neighbourhood level network, there are different types of power consumption: EV charging power consumption, base load power consumption and home based batteries power consumption. The charging, discharging of EVs, home based batteries and power consumption for controllable home appliances could be controlled by the central controller.

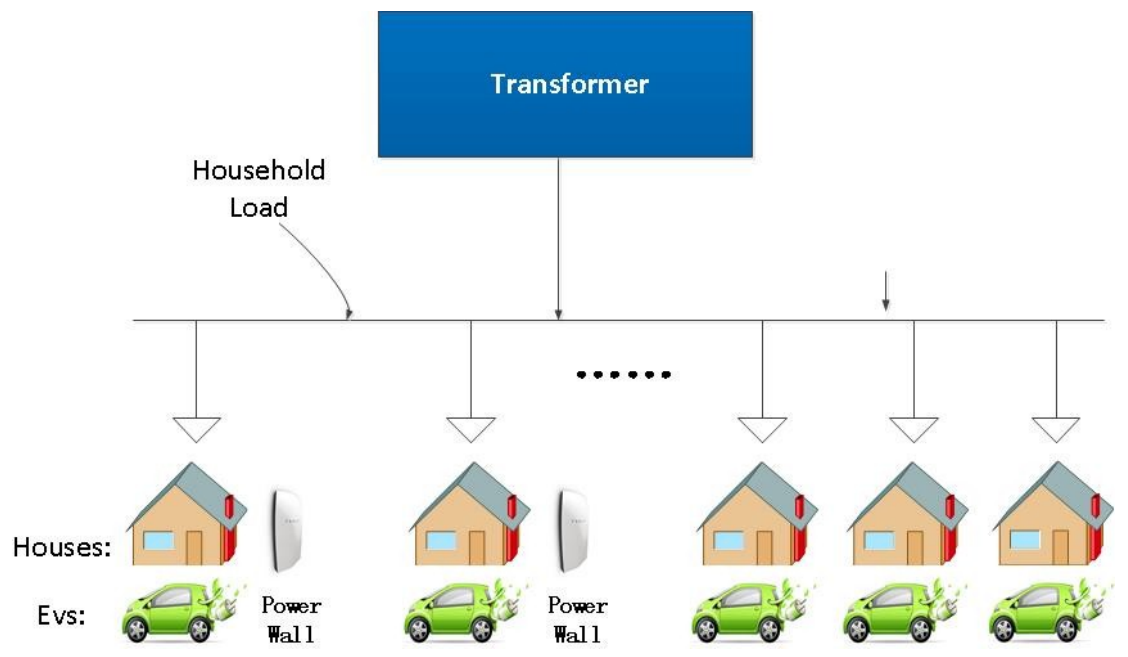

Figure 2: neighbourhood level network

\subsection{Time-of-Use price}

Additional power generation will be needed for peak power consumption hours which will be very expensive. In order to encourage people to shift their power consumption to valley hours, time-of-use price structure is adopted by some utility companies. Under time-of-use price structure, the electricity price will be high for peak hours and low for valley hours. 


\section{EV Charging Control Framework}

\subsection{Objective function}

The high peak power consumption may negatively affect the network and the electricity cost. In this paper, we propose a centralized control framework for the control of EV charging, controllable home appliances and home based batteries. The objective function is to minimize peak power consumption as shown in Equation (1). The peak power consumption shows the discrete-time maximal power consumption in one day. $p_{b}$ shows the total base load power consumption. $p_{e v c}$ is the total EV charing power consumption. $p_{e v c d}$ is the total EV discharging power consumption. $p_{b a}$ is total charging consumption for home based batteries, $p_{b a d}$ is the total discharging power consumption for home based batteries. $p_{\text {reu }}$ shows the used renewable energy.

$$
\operatorname{Min}\left\{p_{g \_m a x}=\operatorname{Max}\left(p_{b}[t]+p_{\text {evc }}[t]+p_{b a}[t]-p_{\text {bad }}[t]-p_{\text {evcd }}[t]-p_{\text {reu }}[t]\right)\right\}, t \in[1,24]
$$

\subsection{Constraints}

The following system constraints should be satisfied in all time slots. These constraints include power balance constraint, total power consumption constraint, charging timing constraint and renewable energy constraint. These constraints are discussed in the following equations. There are two kinds of constraints: inequality constraints and equality constraints.

\section{1) Power balance constraint:}

Equation (2) means that for every time slot, the power supply side and consumption side should be balanced. The left side shows power supply side including: power from power system $\left(p_{g}\right)$, discharging from home based batteries $\left(p_{b a d}\right)$, discharging from EVs $p_{\text {evcd. }}$. The right side in equation (2) shows power consumption side, including: base load power consumption $p_{b}$, EV charging power consumption $p_{e v c}$, and home based batteries charging power consumption $p_{b a}$.

$$
p_{g}[t]+p_{b a d}[t]+p_{\text {evcd }}[t]=p_{b}[t]+p_{\text {evc }}[t]+p_{b a}[t], \forall t
$$

\section{2) Charging time constraint}

Equation (3) and (4) show the timing constraint for every electric vehicle. The EVs can only be charged or discharged with controlled rate only when these EVs are parked homes. Here $p_{\text {evci }}[i][t]$ and $p_{\text {evcdi }}[i][t]$ show the EV charging and discharging power consumption for $i^{t h} \mathrm{EV}$ at time slot $t . t c[i][t]$ shows the timing constraint for $i^{t h} \mathrm{EV}$ at time slot $t$. If $\mathrm{EV}$ is parked in the home for time slot $t$, the $t c$ will be equal to the maximal rated charging power for EV.

$$
\begin{aligned}
& p_{\text {evci }}[i][t] \leq t c[i][t], \forall t \\
& p_{\text {evcdi }}[i][t] \leq t c[i][t], \forall t
\end{aligned}
$$

3) Controllable base load power consumption constraint

Function 5 shows that there are two parts of base load power consumption $\left(p_{b}\right)$ : controllable base load power consumption $\left(p_{b c}\right)$, and uncontrollable base load power consumption $\left(p_{b u}\right)$.

$$
p_{b}[i][t]=p_{b c}[i][t]+p_{b u}[i][t], \forall t
$$

\section{4) Battery state of charge constraint}

Equation (6) and (7) shows the battery state of charge requirement for EVs. EVs should be charged with enough energy before the departure time. bsoc $[i]$ shows the final battery state of charge, bsoci $[i]$ shows the initial battery state of charge for $i^{\text {th }}$ electric vehicle. cap $[i]$ shows the EV battery capacity for $i^{\text {th }} \mathrm{EV}$. $b_{s o c}[i]$ shows the required battery state of charge for $i^{\text {th }}$ electric vehicle.

$$
\begin{gathered}
b \operatorname{ssoc}[i]=b \operatorname{soc} i[i]+\sum_{j=1}^{M}\left(p_{\text {evci }}[i][t]-p_{\text {evcdi }}[i][t]\right) \frac{1}{\operatorname{cap}[i]} \cdot 100 \\
\quad \operatorname{bsoc}[i] \geq \text { bsoc }_{\text {req }}[i]
\end{gathered}
$$

5) Renewable energy constraint

Equation (8) shows that the renewable energy $\left(p_{\text {reu }}[t]\right)$ that could be used should be smaller than the actual generated renewable energy $p_{r e}[t]$ for every time slot.

$$
p_{\text {reu }}[t] \leq p_{\text {re }}[t], \forall t
$$




\section{6) Home based battery constraint}

Equation (9) and (10) show that the home based batteries should be charged with enough energy at the end of every day. $p_{\text {bai }}[i][t]$ and $p_{\text {badi }}[i][t]$ shows the charging and discharging power for home based batter in $i^{\text {th }}$ home at time slot $t$. capba [i] shows the battery capacity for $i^{\text {th }}$ home based battery. babsoc req $[i]$ shows the required battery state of charge for $i^{t h}$ home based battery. basoci $[i]$ means the initial battery of charge for $i^{\text {th }}$ home based battery.

$$
\begin{gathered}
\text { basoc }[i]=\text { basoci }[i]+\sum_{j=1}^{24}\left(p_{\text {bai }}[i][t]-p_{\text {badi } i}[i][t]\right) \frac{1}{\operatorname{capba}[i]} \cdot 100 \\
\text { basoc }[i] \geq \text { babsoc }_{\text {req }}[i]
\end{gathered}
$$

\section{7) EV charging limit constraint}

Equation (11) and (12) show that for every time slot, the charging power and discharging for every electric vehicle should be smaller than the maximal rated charging power $p_{\text {evi_max }}[i]$.

$$
\begin{gathered}
p_{\text {evc }}[i][t] \leq p_{\text {evi_max }}[i], \forall t \\
p_{\text {evcdi }}[i][t] \leq p_{\text {evi_max }}[i], \forall t
\end{gathered}
$$

Equation (13) and (14) show that for every time slot, the charging power and discharging for the home based batteries should be smaller than the rated maximal rated charging power $p_{b a \_m a x}[i]$.

$$
\begin{gathered}
p_{b a}[i][t] \leq p_{\text {ba_max }}[i], \forall t \\
p_{\text {baci }}[i][t] \leq p_{\text {ba_max }}[i], \forall t
\end{gathered}
$$

\section{8) Total charging and discharging constraint}

We assume that there are $\mathrm{N}$ electric vehicles in the neighbourhood level network and there is one electric vehicle and home based battery in every home. Equation (15) and equation (16) show the total charging constraint $\left(p_{\text {evc }}[t]\right)$ and total discharging constraint $\left(p_{\text {evcd }}[t]\right)$ for every time slot.

$$
\begin{gathered}
p_{\text {evc }}[t]=\sum_{i=1}^{N} p_{\text {evci }}[i][t], \forall t \\
p_{\text {evcd }}[t]=\sum_{i=1}^{N} p_{\text {evcdi }}[i][t], \forall t
\end{gathered}
$$

Equation (17) and equation (18) show the total charging power for home based batteries $\left(p_{b a}[t]\right)$ and total discharging power for home based based batteries $\left(p_{\text {bad }}[t]\right)$ for every time slot.

$$
\begin{gathered}
p_{b a}[t]=\sum_{i=1}^{N} p_{b a i}[i][t], \forall t \\
p_{b a d}[t]=\sum_{i=1}^{N} p_{\text {badi }}[i][t], \forall t
\end{gathered}
$$

\section{Simulation Results}

In this section, we use the proposed control framework to study the impacts for integration control of EV charging, controllable home appliances and home based batteries on the peak power consumption on the neighbourhood level network. The framework presented in Section 3 is modeled in Java and solved with the server CPLEX v.12. Simulation results are presented in this section.

Here two kinds of electric vehicles are considered: Nissan Leaf and Tesla Model S. As for the Nissan Leaf, the maximal charging power is $6.6 \mathrm{~kW}$ and battery capacity is $24 \mathrm{kWh}$. As for Tesla Model S, the battery capacity of $60 \mathrm{kWh}$ and rated charging power of $10 \mathrm{~kW}$ are adopted. The EVs are required to be charged with enough energy before the departure time. The final battery state of charge of EVs and 
Table 3: Power consumption for controllable home appliances

\begin{tabular}{|c|c|c|}
\hline Controllable Based Load & Daily Energy Consumption & Hourly Power Consumption \\
\hline Washing Machine & $1.6 \mathrm{kWh}$ & $0.8 \mathrm{~kW}$ \\
\hline Dishwasher & $2.4 \mathrm{kWh}$ & $1.2 \mathrm{~kW}$ \\
\hline
\end{tabular}

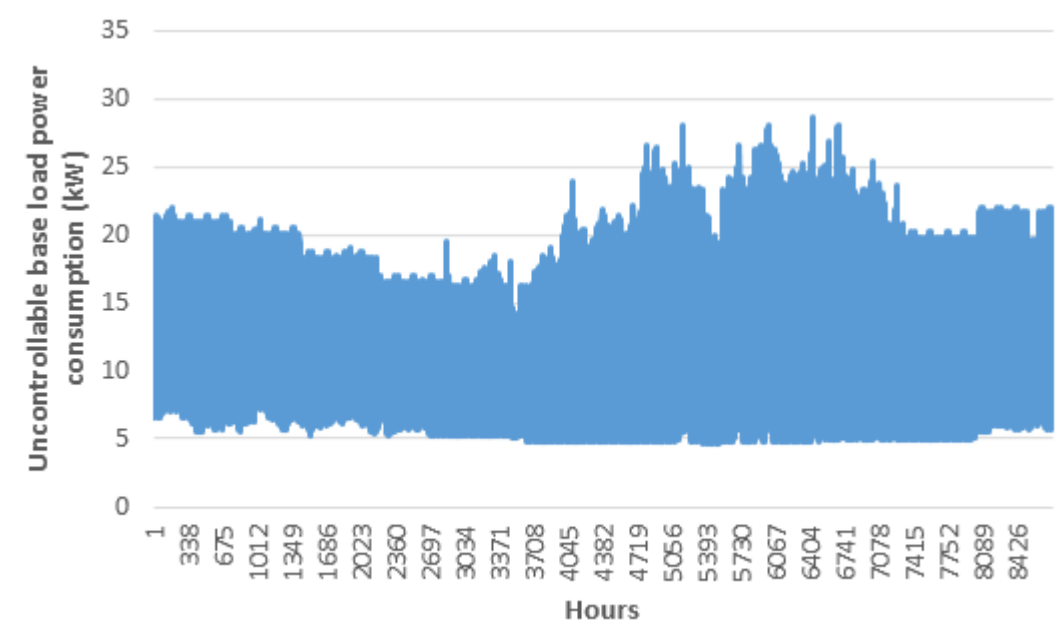

Figure 3: Base load power consumption for one year

home based batteries are required be larger than 90. The arrival time for the EVs are set to be 5 pm, 6 pm, $7 \mathrm{pm}$ with the probability of $(20 \%, 40 \%$ and 40\%). The departure time for the EVs are set to be 7 am, $8 \mathrm{pm}, 9 \mathrm{pm}$ with the probability of $(30 \%, 50 \%$ and $20 \%)$. The arrival battery state of charge for EVs are set to be $30,50,70$ with the probability of $(30 \%, 50 \%$ and $20 \%)$.

We consider that there are six homes in this neighbourhood level network. The data presented in [15] is used as the uncontrollable base load power consumption for one home. We use this data and add the uniform distribution noise of $[-1,1]$ to build the uncontrollable base load power consumption for all the homes in the whole network. Figure 3 shows the one year hourly data for uncontrollable based load power consumption for the network. We assume that there is one electric vehicle in every home. The controllable home appliances includes: washing machine and dishwasher. The power consumptions for controllable base load are shown in Table 3. The default starting time for the controllable appliances for all the homes are set to be at $7 \mathrm{pm}$. The home based batteries is chosen as Tesla Battery Wall. The battery

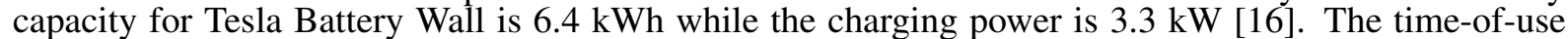
price structure presented in Figure 4 is used for calculating the electricity cost [17]. The rated maximal power for the transformer of the network is chosen as $50 \mathrm{~kW}$.

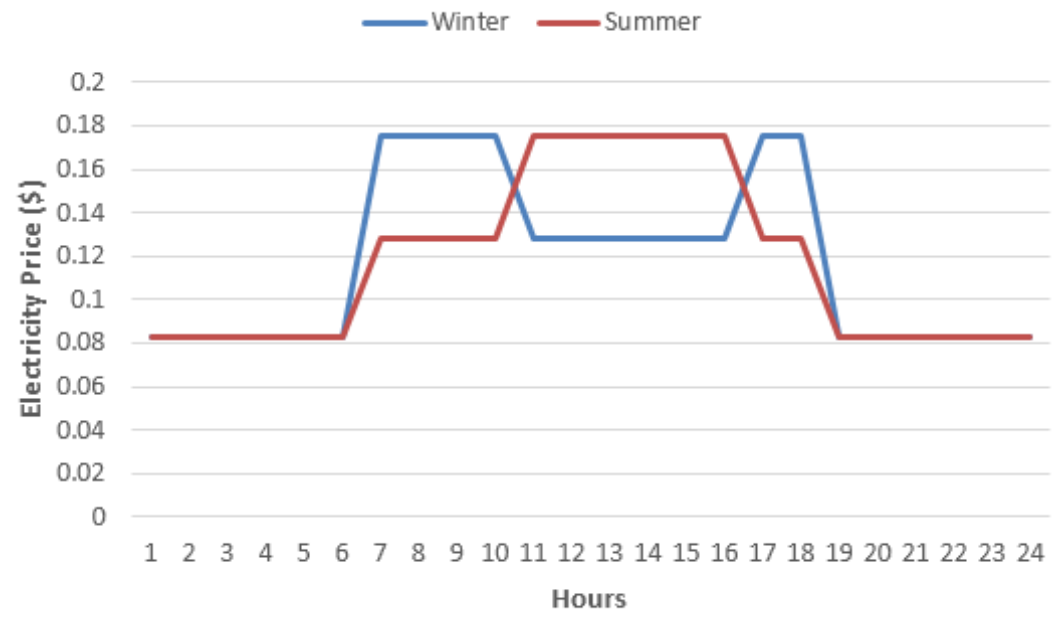

Figure 4: Time-of-use price structure 


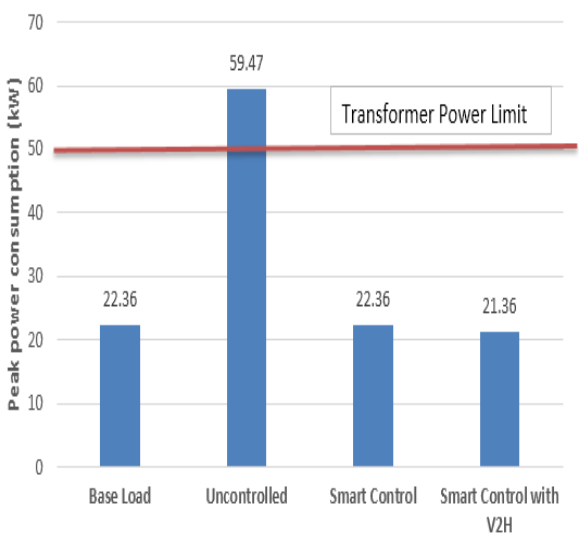

Figure 5: Peak power consumption for a summer day with Nissan Leaf

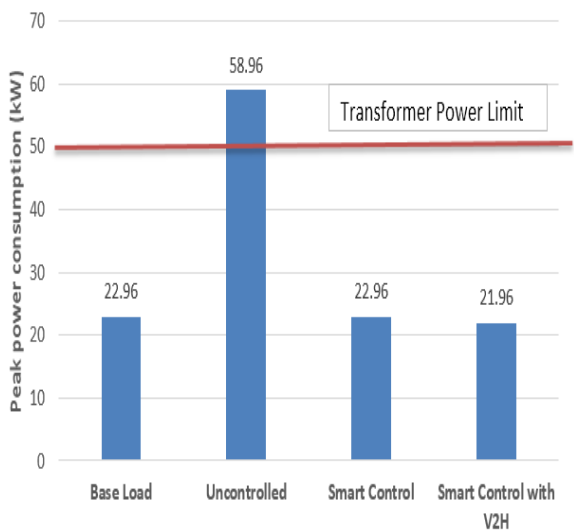

Figure 7: Peak power consumption for a winter day with Nissan Leaf

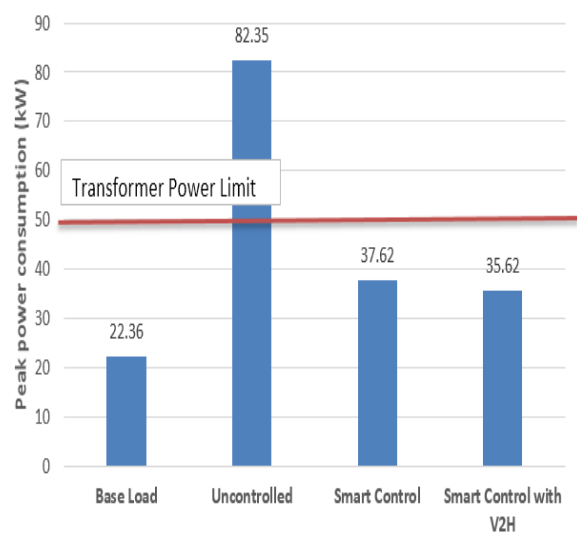

Figure 6: Peak power consumption for a summer day with Tesla Model S

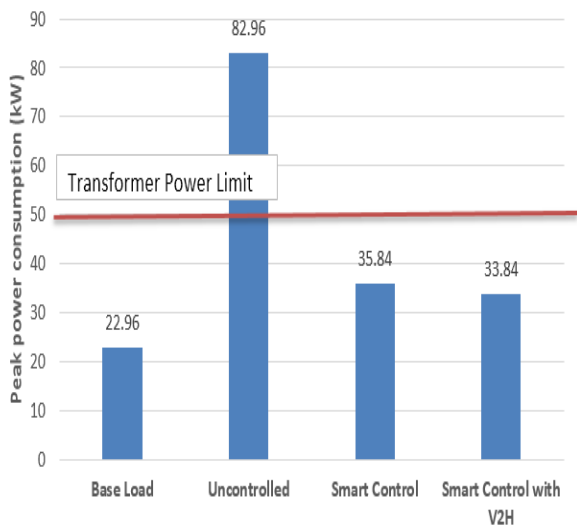

Figure 8: Peak power consumption for a winter day with Tesla Model S

\subsection{Peak power reduction with controlled EV charging}

We studied the peak power consumption of the network for three charging scenarios: uncontrolled EV charging; smart EV charging and smart EV charging with V2H. Uncontrolled EV charging means that the EVs will be charged as soon as they arrive home. Smart EV charging means the EVs are charged with the method proposed in Section 3, but V2H is not considered for this scenario. Smart EV charging with $\mathrm{V} 2 \mathrm{H}$ means that we further consider that the energy stored in EVs could be further discharged back for residential consumption. The controllable appliances start to work at default time and home based batteries are not considered in this section. Simulation results for one typical summer day for Nissan Leaf and Tesla Model S are shown in Figure 5 and Figure 6. Simulation results for one typical winter day for Nissan Leaf and Tesla Model S are shown in Figure 7 and Figure 8. We can see that without any control the peak power consumption will exceed the transformer power limit. This will be more server for Tesla Model $\mathrm{S}$ which will require more charging power. Simulation results show that with smart control for EV charging, the peak power consumption could be significantly reduced compared with uncontrolled charging for both Nissan Leaf and Tesla Model S. It also shows that when V2H is adopted the peak power consumption could be further reduced. Smart EV charging control could help to minimize the peak power consumption and protect the power system.

\subsection{Peak power reduction with controlled home appliances and battery}

Here the EVs are charged with smart charging and V2H is not adopted. Figure 9 shows the peak power consumption of the network for a summer day with Nissan Leaf. Simulation results for five scenarios are shown: base load power consumption with no EVs; smart charging control; smart charging control with 
one home based battery; smart charging control with two home based batteries; smart charging control with two home based batteries and adopt home appliance control. The simulation results for Tesla Model $\mathrm{S}$ is shown in Figure 10.

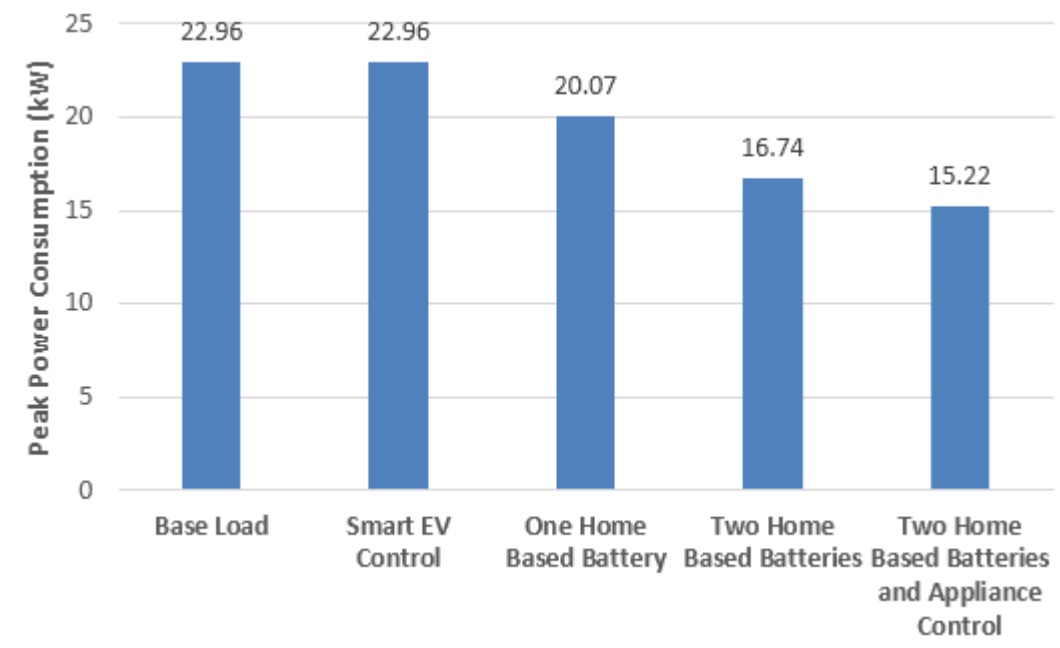

Figure 9: Peak power consumption for a typical summer day with Nissan Leaf

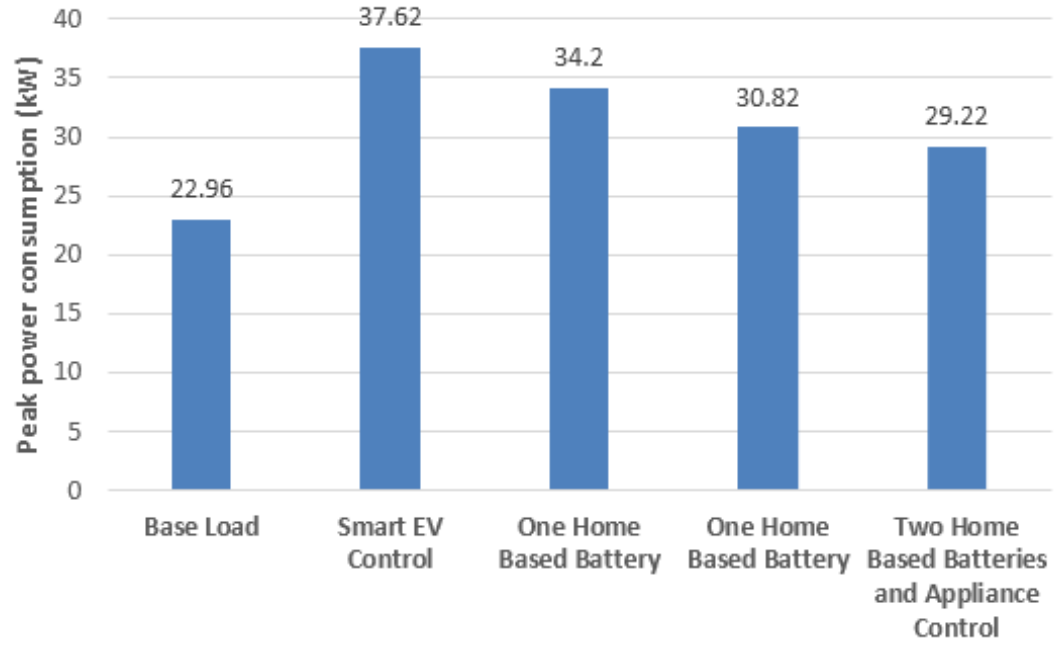

Figure 10: Peak power consumption for a typical summer day with Tesla Model S

From the simulation results we can see that, when the home based batteries are adopted the peak power consumption of the network could be significantly reduced. Battery wall can store energy during the valley hours and discharge the energy back for the peak hours. In this paper, we consider that the controllable home appliances like: washing machine and dishwasher could be controlled by the system central controller. These appliances could be controlled discretely, which means that we can decide the on and off of these appliances but the for a certain time slot the power consumption will be a fixed value. We can see that when the control for home appliance is adopted the peak power consumption for the network could be further reduced.

\section{Conclusion}

In this paper, an EV charging framework is proposed to minimize the peak power consumption. The control framework is used to study the peak power consumption for different scenarios. Based on the simulation results we can see that without any control the peak power consumption of the network could easily exceed the transformer power limit. The adoption of EV charging control and home based batteries could help significantly reduce the peak power consumption. The $\mathrm{V} 2 \mathrm{H}$ and control of controllable home 
appliance could help to further reduce the peak power consumption. These techniques could help the power system to handle more EVs and protect the network infrastructures. In the future work, we will study the impact of such integration control on the electricity cost for the neighbourhood level network.

\section{References}

[1] https://www3.epa.gov/climatechange/ghgemissions/sources/transportation.html, "United states environmental protection agency."

[2] http://www.worldwatch.org/node/6251, "German government, electromobility in germany: Vision 2020 and beyond. report 1 (2013)."

[3] http://www.irena.org/DocumentDownloads/Publications/IRENA_REmap_Germany_report_2015.pdf, "Renewable energy prospects: Germany."

[4] E. Coalition, "electrification roadmap revolutionizing transportation and achieving energy security," http://www.electrificationcoalition.org/policy/electrification-roadmap.

[5] M. H. Tushar, C. Assi, M. Maier, and M. F. Uddin, "Smart microgrids: Optimal joint scheduling for electric vehicles and home appliances," Smart Grid, IEEE Transactions on, vol. 5, no. 1, pp. 239-250, 2014.

[6] A. Alahyari, M. Fotuhi-Firuzabad, and M. Rastegar, "Incorporating customer reliability cost in pev charge scheduling schemes considering vehicle-to-home capability," Vehicular Technology, IEEE Transactions on, vol. 64, no. 7, pp. 2783-2791, 2015.

[7] F. Berthold, B. Blunier, D. Bouquain, S. Williamson, and A. Miraoui, "Phev control strategy including vehicle to home (v2h) and home to vehicle (h2v) functionalities," in Vehicle Power and Propulsion Conference (VPPC), 2011 IEEE. IEEE, 2011, pp. 1-6.

[8] Z. Wang and G. Zheng, "Residential appliances identification and monitoring by a nonintrusive method," Smart Grid, IEEE Transactions on, vol. 3, no. 1, pp. 80-92, 2012.

[9] J. Zhao, S. Kucuksari, E. Mazhari, and Y.-J. Son, "Integrated analysis of high-penetration pv and phev with energy storage and demand response," Applied Energy, vol. 112, pp. 35-51, 2013.

[10] O. Erdinc, N. G. Paterakis, T. D. Mendes, A. G. Bakirtzis, and J. P. Catalão, "Smart household operation considering bi-directional ev and ess utilization by real-time pricing-based dr," Smart Grid, IEEE Transactions on, vol. 6, no. 3, pp. 1281-1291, 2015.

[11] S. Shao, M. Pipattanasomporn, and S. Rahman, "Challenges of phev penetration to the residential distribution network," in Power \& Energy Society General Meeting, 2009. PES'09. IEEE. IEEE, 2009, pp. 1-8.

[12] A. D. Hilshey, P. D. Hines, P. Rezaei, and J. R. Dowds, "Estimating the impact of electric vehicle smart charging on distribution transformer aging," Smart Grid, IEEE Transactions on, vol. 4, no. 2, pp. 905-913, 2013.

[13] http://www.nissanusa.com/electric-cars/leaf/charging range/, "Nissanusa website."

[14] https://www.teslamotors.com/models charging\#/basics, "Teslamotors website."

[15] http://en.openei.org/doe-opendata/dataset/commercial-and-residential-hourly-load-profiles-for-alltmy3-locations-in-the-united states, "Us department of energy, open data catalog."

[16] https://www.teslamotors.com/powerwall, "Teslamotors website."

[17] https://hydroottawa.com/accounts-and-billing/residential/rates-and conditions, "Hydroottawa." 


\section{Authors}

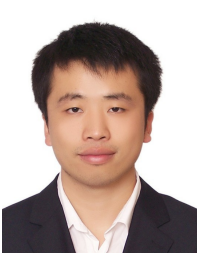

Di Wu is a PhD student in McGill University, and his research fields lie in optimization for electric vehicle charging scheduling and stochastic optimization. Di Wu obtained the bachelor degree from Hefei University of Technology in 2010, and master degree from Peking University in 2013, both are in Microelectronics. Now he is pursing his PhD degree in McGill University.

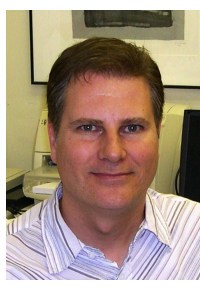

Benoit Boulet, Eng., Ph.D., SMIEEE, is Associate Dean (Research \& Innovation) of the Faculty of Engineering at McGill University and an Associate Professor in the Department of Electrical and Computer Engineering. Professor Boulet obtained a Bachelor's degree from Universit Laval in 1990, a Master of Engineering degree from McGill University in 1992, and a Ph.D. degree from the University of Toronto in 1996, all in electrical engineering. He is a registered Professional Engineer in the province of Qubec. Professor Boulet's research areas include the control of biomedical systems, green energy systems, robust industrial process control and robust vehicle control.

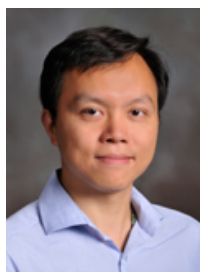

Haibo Zeng is an assistant Professor with the Department of Electrical and Computer Engineering. His research interests include design methodology, analysis and optimization for embedded systems, real-time systems, and cyber physical systems. 